\title{
Efectos de cultivos de cobertura sobre el agua del suelo, el aporte de carbono al sistema y el rendimiento de soja en diferentes ambientes
}

\author{
Capurro, J.E. ${ }^{1} \&$ Montico, S. ${ }^{2}$
}

\begin{abstract}
RESUMEN
La sustentabilidad de los ambientes agrícolas en la región pampeana húmeda argentina es afectada por el monocultivo de soja (Glycine max (L.) Merr.). La baja eficiencia en el uso de los recursos del ambiente durante los barbechos y su efecto negativo sobre la sostenibilidad y productividad de los sistemas de producción hace necesaria la adopción de prácticas tales como la inclusión de un cultivo de cobertura (CC) invernal entre los cultivos de renta estivales.

Se evaluó en una secuencia soja-soja el efecto de un cultivo de cobertura de avena (Avena sativa L.) y vicia (Vicia sativa L.) sobre el aporte de carbono (C) a través de la materia seca (MS) producida, el agua útil disponible del suelo (AUD) y el rendimiento del cultivo de soja sucesor, en distintos ambientes diferenciados por la pendiente. Los tratamientos con CC (CCC) presentaron más AUD en siembra y floración de soja, mayores rendimientos de soja y mayores aportes de $\mathrm{C}$ al sistema.
\end{abstract}

Palabras clave: monocultivo de soja, cultivos de cobertura, sustentabilidad.

1.- AER INTA Cañada de Gómez. Lavalle 1466 (2500) Cañada de Gómez, provincia de Santa Fe, Argentina. Emial: capurro.julia@inta.gob.ar

2.- Cátedra Manejo de Tierras FCA, UNR. CC No 14 (2125) Bv. Villarino, Zavalla, provincia de Santa Fe, Argentina.

Manuscrito recibido el 18 de mayo de 2021 y aceptado para su publicación el 6 de diciembre de 2021.

Capurro, J.E. \& Montico,S. Efectos de cultivos de cobertura sobre el agua del suelo, el aporte de carbono al sistema y el rendimiento de soja en diferentes ambientes. FAVE - Ciencias Agrarias 21 (1): 49-65 CC BY-NC-SA 4.0 


\begin{abstract}
Effects of cover crops on soil water, the supply of carbon to the system and the yield of soybean in different environments.

The sustainability of agricultural environments in the Humid Argentine Pampean Region is affected by the monoculture of soybean (Glycine max (L.) Merr.). The low efficiency in the use of environmental resources during fallows and its negative effect on the sustainability and productivity of production systems makes it necessary to adopt practices such as the inclusion of a winter cover crop (CC) between the income summer crops. In a soybean-soybean sequence, the effect of a cover crop of oats (Avena sativa L.) and vicia (Vicia sativa L.) on the contribution of carbon (C) through the dry matter (DM) produced, the available soil water (AUD) and the yield of the subsequent soybean crop were evaluated, considering environments differentiated by the slope. The treatments with CC (CCC) presented more AUD in soybean planting and flowering, higher soybean yields and greater contributions of $\mathrm{C}$ to the system.
\end{abstract}

Key words: monoculture, cover crops, sustainability.

\section{INTRODUCCIÓN}

Las secuencias de soja (Glycine max $L$. Merr.) continua, que ocupan actualmente una gran superficie del área cultivada en la región pampeana húmeda argentina, afectan la productividad y sustentabilidad de esos ambientes. El sistema de producción actual, basado en una agricultura continua bajo siembra directa donde prevalecen los cultivos de verano, suele dejar un período de tiempo el suelo descubierto, normalmente durante el otoño e invierno, el cual se puede extender de 5 a 9 meses (Novillo et al., 2018). En los suelos de ambientes ondulados, esta condición se incrementa dado que el agua de los escurrimientos superficiales, arrastra los rastrojos hacia las zonas más bajas de los lotes. Por otra parte, una gran proporción de la oferta anual de recursos del ambiente útiles para la productividad agrícola, principalmente agua y radiación solar, son desaprovechados al realizar un solo cultivo por año, lo cual torna en sumamente ineficientes a los actuales sistemas productivos (Caviglia et al., 2004). La evaporación es el principal factor de pérdida de agua de los suelos (Quiroga et al., 2005) estimándose que entre el $50 \%$ y 70 \% de la precipitación anual retorna directamente a la atmósfera sin intervenir en el proceso productivo. La pérdida de agua por evaporación desde la superficie del suelo, puede reducirse con coberturas vivas como CC, que al aumentar la transpiración reasignan el agua potencialmente evaporada al flujo transpiratorio de las plantas (Lu et al., 2000 citado por Carfagno et al., 2013a). El uso de CC podría mejorar entre otras propiedades del suelo, la captura de agua de lluvia en comparación con los barbechos largos encontrados en los sistemas de monocultivo de soja, contribuyendo a la adaptación al incremento en la intensidad de lluvias primaverales, asociado al cambio climático (Melgares et al. 2018). 
Los CC se siembran en la Región Pampeana Argentina, mayoritariamente, luego de la cosecha de un cultivo de verano y se suprime su crecimiento en forma química o mecánica, antes de la siembra del cultivo estival siguiente. También pueden ser aprovechados parcialmente como forrajes de doble propósito, en sistemas de producción mixtos o integrados. De esta forma se incorpora $\mathrm{C}$ al sistema, se utilizan insumos del ambiente que hubieran sido desaprovechados, se exploran a través de sus raíces sectores compactados por la maquinaria agrícola y se aumenta la cobertura superficial. Cuando el CC utilizado es una leguminosa, se logra además un ingreso adicional de nitrógeno $(\mathrm{N})$, lo que puede representar una ventaja económica agregada (Ernst, 2004). Esto se da, generalmente, cuando se realiza una adecuada inoculación de la semilla de la leguminosa. La cobertura del suelo proporcionada por los residuos de cultivos dejados en superficie (Siqueira Leite et al., 2009) tiene acción directa y efectiva en la reducción de la erosión hídrica, en virtud de la disipación de la energía cinética de las gotas de lluvia, lo cual disminuye la desagregación de las partículas del suelo y el sellamiento superficial y aumenta la infiltración del agua.

La incorporación de un CC invernal en la secuencia soja-soja, podría ocasionar variaciones de la productividad del cultivo de cosecha, según los tipos (presencia o ausencia de pendiente) y la calidad (definida por sus propiedades físicas, químicas y biológicas) de los ambientes donde se realicen. Este trabajo tuvo como propósito evaluar el efecto de la de la secuencia CC-soja, en el almacenaje de agua del suelo, en el aporte de materia seca y $\mathrm{C}$, y en algunos componentes de rendimiento del cultivo de soja, en distintos ambientes diferenciados por la pendiente.

\section{MATERIALES Y MÉTODOS}

El estudio se realizó sobre un lote con manejo de agricultura continua durante 25 años y monocultivo de soja de primera en siembra directa, durante los últimos diez años, en Cañada de Gómez, provincia de Santa Fe, República Argentina ( $32^{\circ}$ 52' 33”' Sur y 61 $26^{\circ}$ ' 59”' Oeste, altitud media de $114 \mathrm{~m}$ ), durante el período 2012/2013. El suelo es un Argiudol típico serie Correa (horizonte superficial 0-16 cm: arcilla 23,5\%; arena 7,0\%; limo 69,5\%).

Según la Clasificación Climática de Thornthwaite (Thornthwaite y Mather, 1955), el clima de la región es de tipo subhúmedo a húmedo. El régimen pluviométrico tiende a ser monzónico, con un 70 \% de las lluvias concentradas en el verano, de octubre a marzo. La temperatura media anual es de $17,5^{\circ} \mathrm{C}$, con una máxima media de $23,5^{\circ} \mathrm{C}$ y una mínima media de $12,3^{\circ} \mathrm{C}$. La humedad relativa media anual es de $74 \%$ y la frecuencia media de días con heladas es de 16 por año, que se producen desde inicios del mes de junio hasta inicios del mes de septiembre. En la Figura 1 se muestran los valores medios de precipitaciones en Cañada de Gómez (datos INTA AER Cañada de Gómez, no publicados), correspondientes al período 1957/2012 y los registros de las lluvias caídas durante el período de estudio.

En el momento de siembra de los CC (mayo 2012) se extrajeron muestras compuestas de suelo a $0-5 \mathrm{~cm}, 5-10 \mathrm{~cm}, 10-20$ cm y 20-40 cm de profundidad. Sobre las mismas se determinaron (Tabla 1) las concentraciones de: COT (carbono orgánico total) (g. $\left.\mathrm{kg}^{-1}\right)$ por combustión húmeda según el método de Walkley y Black (1934), Nt (nitrógeno total) (g.kg-1) según el método de Kjeldhal (1883), Pe (fósforo extractable) (mg.kg-1) por el método Bray I (Bray 


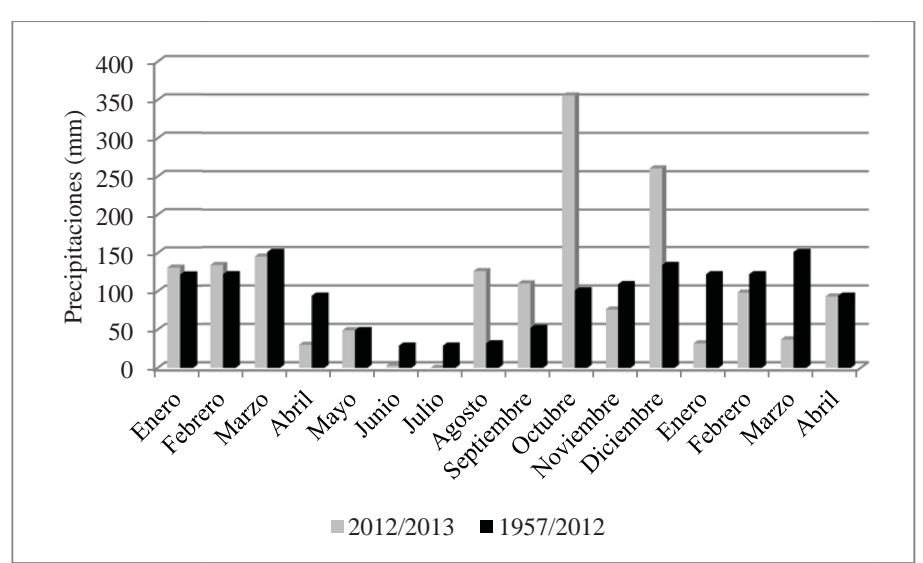

Figura 1. Precipitaciones medias en milímetros del período 1957/2012 y del período enero 2012/abril 2013. Figure 1. Average rainfall in millimeters for the period 1957/2012 and of the period January 2012 / April 2013.

y Kurtz, 1945), S-SO4 (azufre de sulfatos) (mg.kg-1) por el método turbidimétrico y la reacción del suelo $(\mathrm{pH})$ por el método suelo/agua: 1/ 2,5. La relación carbono/nitrógeno $(\mathrm{C} / \mathrm{N})$ se obtuvo mediante el cociente entre $\mathrm{COT} / \mathrm{Nt}$.

El experimento se estableció en un diseño en parcelas divididas, en donde las parcelas mayores correspondieron al factor ambiente con tres niveles: Loma (pendiente: 0,15\%), Media Loma (pendiente: $0,90 \%$ ) y Bajo (pendiente: $0,10 \%$ ) y las parcelas menores correspondieron al sub-factor tratamiento, con dos niveles: CCC (con cultivos de cobertura) y SCC (sin cultivos de cobertura). De esta forma, quedaron definidas seis combinaciones de ambientes y tratamientos: Loma SCC, Loma CCC, Media Loma SCC, Media Loma CCC, Bajo SCC y Bajo CCC. Las dimensiones de cada parcela fueron de $30 \mathrm{~m}$ por $50 \mathrm{~m}$, y se distribuyeron en bloques completos aleatorizados con tres repeticiones.

Manejo del CC: En pre-siembra del CC se eliminaron las malezas emergidas en todas las parcelas con una aplicación de 1,35 kg.ha- ${ }^{-1}$ de Glifosato SG 74,7 \% (679 g de equivalente ácido). La siembra del CC se realizó el 8 de mayo de 2012 con una sembradora de siembra directa de grano fino, con líneas de siembra separadas a 0,175 m y abresurcos de doble disco plano. Se implantó una mezcla de avena (Avena sativa L.) y vicia (Vicia sativa L.) con una densidad de siembra de $88 \mathrm{~kg} \cdot \mathrm{ha}^{-1}$, a fin de lograr 200 plantas por metro cuadrado (pls.m ${ }^{-2}$ ) y una relación de consociación 50:50 de cada especie. La semilla de vicia fue inoculada con Rhizobium leguminosarum bv.viciae, con una dosis de $110 \mathrm{~g}$ de inoculante cada $25 \mathrm{~kg}$ de semilla. El ciclo de crecimiento del CC fue de 155 días, desde la emergencia hasta la supresión de su crecimiento. El 13 de octubre, en estadio de plena floración del CC (vicia 70\%; avena 100\%) se aplicaron 2,5 l.ha ${ }^{-1}$ de Glifosato SL 48\% (360 g de equivalente ácido) y 0,7 1.ha-1 de 2,4 D sal dimetilamina (60 g de equivalente ácido) para efectuar el secado de las plantas. La aplicación se realizó con un equipo pulverizador autopropulsado con pastillas tipo cono hueco, presión de trabajo de 8 bares y 100 
Tabla 1. Propiedades químicas edáficas en los estratos 0 a 5, 5 a 10, 10 a 20 y 20 a 40 centímetros de profundidad, en el momento de siembra de los cultivos de cobertura.

Table 1. Edaphic chemical properties in strata 0 to 5, 5 to 10, 10 to 20 and 20 to 40 centimeters deep, at the time of planting the cover crops.

\begin{tabular}{|c|c|c|c|c|c|c|}
\hline Ambiente & $\begin{array}{c}\text { COT } \\
\left(\mathrm{g} \cdot \mathrm{kg}^{-1}\right)\end{array}$ & $\begin{array}{c}\mathrm{Nt} \\
\left(\mathrm{g} \cdot \mathrm{kg}^{-1}\right)\end{array}$ & C:N & $\begin{array}{c}\mathrm{Pe} \\
\left(\mathrm{mg} \cdot \mathrm{kg}^{-1}\right)\end{array}$ & $\begin{array}{c}\text { S-SO4 } \\
\left(\mathrm{mg} \cdot \mathrm{kg}^{-1}\right)\end{array}$ & $\mathrm{pH}$ \\
\hline \multicolumn{7}{|c|}{$0-5 \mathrm{~cm}$} \\
\hline Loma & 24,03 & 1,90 & 12,65 & 37,43 & 9,80 & 6,17 \\
\hline $\begin{array}{l}\text { Media } \\
\text { Loma }\end{array}$ & 20,00 & 1,63 & 12,27 & 16,93 & 11,37 & 5,93 \\
\hline Bajo & 22,02 & 1,73 & 12,73 & 40,93 & 10,40 & 6,00 \\
\hline \multicolumn{7}{|c|}{$5-10 \mathrm{~cm}$} \\
\hline Loma & 16,55 & 1,40 & 11,82 & 22,87 & 10,57 & 5,53 \\
\hline $\begin{array}{l}\text { Media } \\
\text { Loma }\end{array}$ & 14,63 & 1,23 & 11,89 & 10,13 & 8,13 & 5,77 \\
\hline Bajo & 15,39 & 1,37 & 11,23 & 22,50 & 9,73 & 5,80 \\
\hline \multicolumn{7}{|c|}{$10-20 \mathrm{~cm}$} \\
\hline Loma & 16,90 & 1,47 & 11,50 & 21,50 & 11,60 & 5,40 \\
\hline $\begin{array}{l}\text { Media } \\
\text { Loma }\end{array}$ & 14,23 & 0,97 & 14,67 & 9,27 & 8,63 & 5,67 \\
\hline Bajo & 16,44 & 1,30 & 12,65 & 24,50 & 9,13 & 5,60 \\
\hline \multicolumn{7}{|c|}{$20-40 \mathrm{~cm}$} \\
\hline Loma & 15,06 & 1,17 & 12,87 & 18,83 & 7,40 & 5,93 \\
\hline $\begin{array}{l}\text { Media } \\
\text { Loma }\end{array}$ & 11,24 & 0,87 & 12,92 & 8,70 & 8,77 & 6,07 \\
\hline Bajo & 12,87 & 1,07 & 12,03 & 24,20 & 7,73 & 5,87 \\
\hline
\end{tabular}

1.ha ${ }^{-1}$ de caudal de agua. Las precipitaciones registradas durante el ciclo de crecimiento de los CC sumaron $391 \mathrm{~mm}$.

Manejo del cultivo de soja: La siembra del cultivo de soja se efectuó el 10 de noviembre, con una sembradora de siembra directa de grano grueso, con líneas de siembra separadas a $0,525 \mathrm{~m}$, cuchillas turbo, doble disco plantador y placas monograno. Se implantó un cultivar de grupo de madurez IV corto indeterminado a una densidad de 34 semillas por metro cuadrado (18 semillas por metro lineal a 0,525 m entre líneas de siembra). La semilla de soja fue inoculada con Bradyrhizobium japonicum, con una dosis de $200 \mathrm{~g}$ de inoculante cada $50 \mathrm{~kg}$ de semilla y tratada con curasemillas. La cosecha del cultivo de soja se realizó el 7 de abri de 2013 con una cosechadora autopropulsada. Las precipitaciones registradas durante el ciclo de la soja totalizaron $497 \mathrm{~mm}$.

Determinaciones realizadas: En forma previa al secado de los CC, con las plantas en el estadio de plena floración, se extrajeron tres muestras de 0,25 metro cuadrado $\left(\mathrm{m}^{2}\right)$ de la parte aérea del CC en cada parcela. Se obtuvo el peso fresco, se llevó a estufa a $65{ }^{\circ} \mathrm{C}$ hasta peso constante y se obtuvo el peso seco. Se calculó la producción de MS en Mg.ha-1 ${ }^{-1}$ Se determinó la concentración de carbono en porcentaje (\% C) con analizador de carbono por combustión seca (LECO-TRUSPEC). Se calculó el conteni-

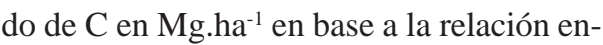
tre la producción de MS y la concentración de $\mathrm{C}$ en los tejidos vegetales. 
En cinco momentos del estudio se midió la lámina de agua actual en $\mathrm{mm}$, hasta dos metros de profundidad, con una sonda de neutrones marca Troxler. Luego, en base a las constantes hídricas - punto de marchitez permanente (PMP) y capacidad de campo (CC) - del suelo del sitio experimental, se calculó el agua útil disponible (AUD) en $\mathrm{mm}$. Las determinaciones se realizaron en siembra de los CC, secado de los CC, siembra de soja, inicio de floración de soja (R1) e inicio de llenado de granos de soja (R5). En el cultivo de soja -en estadio R6se extrajeron 3 muestras de $1 \mathrm{~m}$ lineal de surco de parte aérea de plantas, en cada parcela. Se separaron las hojas y tallos (en adelante Soja 1), de las vainas con granos (en adelante Soja 2) y en ambas fracciones se obtuvo el peso fresco. Se llevó a estufa a $65^{\circ} \mathrm{C}$ hasta peso constante y se obtuvo el peso seco. Se calculó la producción de MS en $\mathrm{Mg}_{\mathrm{g}} \mathrm{ha}^{-1}$. Se determinó la concentración de carbono en porcentaje (\% C) con analizador de carbono por combustión seca (LECO-TRUSPEC). Se calculó el contenido de C en Mg.ha ${ }^{-1}$ en base a la relación entre la producción de MS y la concentración de C en los tejidos vegetales.

Las parcelas se cosecharon con cosechadora automotriz. El grano cosechado se pesó con una balanza portátil para acoplados marca Vesta, de 4 platos. La determinación de la humedad del grano cosechado se realizó con un higrómetro electrónico marca Delver, modelo HD-1000 digital. El rendimiento (kg.ha-1) se llevó a humedad comercial del grano (13,5\%). Se evaluó el peso de mil semillas en g (P1000) y se calculó el número de granos por metro cuadrado (NG).

Los análisis estadísticos se realizaron mediante análisis de variancia testeando particularmente los efectos de ambientes, tratamientos y su interacción. Se realizaron los test de comparaciones de medias, de acuerdo a la significación de los efectos en los análisis de variancia, utilizando el test LSD de Fisher, a un nivel de significación del 5\%. Para el análisis de la variable AUD, observada en distintos momentos, los análisis de variancia se adaptaron a esta situación, creando la variable clasificatoria o efecto TA, constituido por las 6 combinaciones de ambientes y tratamientos y considerando también el efecto fecha y la interacción TAxfecha. Estos análisis se realizaron según un modelo mixto con diferentes matrices de variancias y covariancias. Una vez determinada la estructura adecuada se realizaron los tests de comparaciones de promedios entre fechas, dentro de cada TA. El procesamiento se realizó mediante el software Statistical analysis system (SAS) University edition virtual application (2015).

\section{RESULTADOS Y DISCUSIÓN}

Efecto en la materia seca y carbono de la biomasa aérea de cultivos de cobertura y soja: La producción de MS aérea de los CC, su concentración de C y el contenido resultante del mismo (Tabla 2), no mostraron efecto significativo del ambiente $(\mathrm{p}<$ 0,05 ), a pesar que el ambiente Bajo tuvo una cantidad de biomasa hasta $22 \%$ superior a los otros ambientes. Los registros mostraron incrementos desde la Loma hacia el Bajo, y si bien no fueron significativos, estuvieron posiblemente asociados a un mayor uso consuntivo y a una más alta eficiencia en el uso del agua en el ambiente Bajo (datos no mostrados). 
Tabla 2. Producción de materia seca aérea y contenido de carbono de cultivos de cobertura luego del secado y de soja en madurez fisiológica.

Table 2. Production of aerial dry matter and carbon content of cover crops after drying and of soybeans at physiological maturity.

\begin{tabular}{|c|c|c|c|c|c|c|c|c|c|c|}
\hline \multirow{3}{*}{ Ambiente } & \multirow{3}{*}{ Tratamiento } & \multicolumn{3}{|c|}{ MS (Mg.ha $\left.{ }^{-1}\right)$} & \multicolumn{6}{|c|}{ Carbono } \\
\hline & & \multirow{2}{*}{$\mathrm{CC}$} & \multirow{2}{*}{ Soja1 } & \multirow{2}{*}{ Soja 2} & \multicolumn{2}{|c|}{$\mathrm{CC}$} & \multicolumn{2}{|c|}{ Soja 1} & \multicolumn{2}{|c|}{ Soja2 } \\
\hline & & & & & $\%$ & Mg.ha ${ }^{-1}$ & $\%$ & Mg.ha' ${ }^{-1}$ & $\%$ & Mg.ha ${ }^{-1}$ \\
\hline \multirow{2}{*}{ Loma } & SCC & - & 3238 bc & 5086 bc & - & - & $41,25 a$ & 1336 bc & $43,26 a$ & $2200 \mathrm{~b}$ \\
\hline & $\mathrm{CCC}$ & $5517 \mathrm{a}$ & $4061 a b$ & $5645 \mathrm{a}$ & $39,07 \mathrm{a}$ & $2156 \mathrm{a}$ & $41,53 \mathrm{a}$ & $1686 a$ & $43,61 \mathrm{a}$ & $2462 a$ \\
\hline \multirow{2}{*}{$\begin{array}{l}\text { Media } \\
\text { Loma }\end{array}$} & SCC & - & $3667 \mathrm{abc}$ & $4717 \mathrm{~cd}$ & - & - & $41,62 \mathrm{a}$ & $\begin{array}{c}1526 \\
a b c\end{array}$ & $43,67 \mathrm{a}$ & $2060 \mathrm{~b}$ \\
\hline & $\mathrm{CCC}$ & $5602 a$ & $4116 \mathrm{a}$ & $5575 a b$ & $39,68 \mathrm{a}$ & $2226 a$ & $41,72 \mathrm{a}$ & $1717 \mathrm{a}$ & $44,45 a$ & $2478 a$ \\
\hline \multirow{2}{*}{ Bajo } & $\mathrm{SCC}$ & - & $3004 \mathrm{c}$ & $4508 d$ & - & - & $41,84 \mathrm{a}$ & $1257 \mathrm{c}$ & $43,43 a$ & $1958 \mathrm{c}$ \\
\hline & $\mathrm{CCC}$ & $6734 a$ & $3722 a b$ & $5600 \mathrm{a}$ & $39,11 \mathrm{a}$ & $2634 a$ & $41,98 \mathrm{a}$ & $1561 a b$ & $43,07 \mathrm{a}$ & $2412 a b$ \\
\hline \multirow{2}{*}{$\begin{array}{l}\text { Medias } \\
\text { Trata- } \\
\text { mientos }\end{array}$} & SCC & - & $3303 \mathrm{~b}$ & $4770 \mathrm{~b}$ & - & - & $41,57 \mathrm{a}$ & $1373 \mathrm{~b}$ & $43,46 a$ & $2073 \mathrm{~b}$ \\
\hline & $\mathrm{CCC}$ & 5951 & $3966 \mathrm{a}$ & $5607 \mathrm{a}$ & 39,29 & 2399 & $41,74 \mathrm{a}$ & $1655 a$ & $43,71 \mathrm{a}$ & $2451 \mathrm{a}$ \\
\hline \multirow{3}{*}{$\begin{array}{l}\text { Medias } \\
\text { Ambien- } \\
\text { tes }\end{array}$} & Loma & $5517 \mathrm{a}$ & $3650 \mathrm{a}$ & $5366 \mathrm{a}$ & $39,07 \mathrm{a}$ & $2156 \mathrm{a}$ & $41,39 \mathrm{a}$ & $1511 \mathrm{a}$ & $43,44 a$ & $2331 \mathrm{a}$ \\
\hline & Media Loma & $5602 \mathrm{a}$ & $3892 \mathrm{a}$ & $5146 \mathrm{a}$ & $39,68 \mathrm{a}$ & $2226 a$ & $41,67 \mathrm{a}$ & $1622 \mathrm{a}$ & $44,09 a$ & $2269 \mathrm{a}$ \\
\hline & Bajo & $6734 \mathrm{a}$ & $3363 a$ & $5054 \mathrm{a}$ & $39,11 \mathrm{a}$ & $2634 a$ & $41,91 \mathrm{a}$ & $1409 a$ & $43,23 a$ & $2185 \mathrm{a}$ \\
\hline
\end{tabular}

Referencias: MS: Materia Seca; Soja 1: hojas y tallos en R6; Soja 2: vainas con granos en R6; SCC: Sin Cultivos de Cobertura; CCC: Con Cultivos de Cobertura. Para MS y C de CC: letras distintas en sentido vertical, indican diferencias significativas entre ambientes. Para MS y C de Soja 1 y Soja 2: letras distintas en sentido vertical, indican diferencias significativas entre TA $(p<0,05)$.

References: MS: Dry Matter; Soy 1: leaves and stems in R6; Soy 2: pods with grains in R6; SCC: No Cover Crops; CCC: With Cover Crops. For MS and C of CC: different letters vertically, indicate significant differences between environments. For DM and C of Soy 1 and Soy 2: different letters vertically, indicate significant differences between TA $(p<0.05)$.

Por su parte, la producción de MS aérea del cultivo de soja, su concentración de C y el contenido resultante del mismo (Tabla 2), no mostró interacción de ambiente por tratamiento. En Soja 1 -hojas y tallos- las parcelas CCC superaron significativamente $(\mathrm{p}<0,05)$ a las parcelas SCC, en producción de MS y su consecuente contenido de C. En soja 2 -vainas con granos- también se dio un crecimiento significativo ( $p<$ 0,05 ) de estos valores en las parcelas CCC respecto de las SCC. Los distintos ambientes no presentaron diferencias significativas en estos valores. Estas diferencias significativas pudieron originarse en una mayor reserva de agua del suelo durante las etapas críticas del cultivo -datos presentados más adelante- así como en los mayores contenidos de algunos nutrientes del suelo (datos no mostrados).

$\mathrm{Al}$ adicionar la MS aérea total producida por los CC y la soja, se observa que las parcelas CCC tuvieron incrementos de aportes de la biomasa producida, del 82\% en Loma y Media Loma y del 114\% en Bajo, respecto de las parcelas SCC. En este sentido, Duval et al. (2015) comprobaron que los aportes de $\mathrm{C}$ variaban significativamente al comparar buenas y malas prácticas agrícolas. Así determinaron que el monocultivo 
de soja sólo aportó 54\% del C por hectárea y por año, respecto del que aportaron las prácticas agrícolas que utilizaron CC, rotación de cultivos y fertilización. Igualmente, Caviglia et al. (2013) en base a una serie de experiencias con CC realizadas en el centro oeste de Entre Ríos, concluyeron que los aportes de residuos de los CC y el cultivo de renta siguiente, en todos los casos fueron mayores que los de las alternativas que permanecieron en barbecho durante el invierno.

Efecto en el almacenaje de agua útil disponible en el suelo: El AUD hasta 200 $\mathrm{cm}$ de profundidad, no mostró interacción de ambiente por tratamiento, en ninguna de las cinco fechas evaluadas. Se detectó una interacción de TA por fecha altamente significativa. El análisis entre fechas mostró que, los tratamientos CCC en todos los ambientes, presentaron niveles significativamente superiores de AUD en siembra y floración de soja. En los tratamientos SCC, se dio esta diferencia en siembra de soja, mientras que, en el momento de floración del cultivo, las reservas de AUD habían disminuido significativamente. Además, estos últimos -a excepción de la Loma- tuvieron significativamente más agua en el muestreo que coincidió con el secado en las parcelas CCC.

$\mathrm{Al}$ inicio del estudio, en el momento de siembra de los CC (Figura 2) se halló efecto del ambiente. El Bajo tuvo -con una media de $282 \mathrm{~mm}$ - una disponibilidad de agua útil significativamente superior a los ambientes Loma y Media Loma, que mostraron valores de 185 y 202 mm, respectivamente.

En el momento de secado de la cubierta vegetal (Figura 3), luego de 155 días de crecimiento, se encontró un efecto altamente significativo del tratamiento y sig- nificativo del ambiente. Las parcelas SCC y CCC mostraron una diferencia promedio de AUD de $25 \mathrm{~mm}$ en el perfil del suelo, a favor de las primeras, lo que marcó el consumo de agua de la cubierta invernal. Por su parte, Bajo, Loma y Media Loma mostraron promedios de 294, 258 y $245 \mathrm{~mm}$, respectivamente. La diferencia a favor del Bajo, fue estadísticamente significativa y representó un 14 y 20\% más de AUD que en Loma y Media Loma, respectivamente. En ningún caso, los perfiles estudiados estuvieron por debajo del 78\% del AUM en este momento del estudio.

Durante la siembra del cultivo de soja (Figura 4) luego de 28 días de barbecho, las parcelas SCC y CCC no presentaron diferencias significativas en el AUD $(p<0,05)$, lo que resultó de una mayor capacidad de recarga del agua de lluvia en las parcelas con cubierta vegetal respecto de las parcelas sin cubierta vegetal. El AUD fue de 298, 274 y $260 \mathrm{~mm}$ para los ambientes de Bajo, Loma y Media Loma, favorable significativamente al primero. Todas las parcelas en estudio, mostraron reservas superiores al 85\% del agua útil máxima (AUM).

En el inicio de la floración (R1) del cultivo de soja (Figura 5), los tratamientos SCC y CCC no presentaron diferencias significativas $(p<0,05)$ en el AUD $y$, al igual que en los muestreos anteriores, se halló efecto del ambiente, con una provisión significativamente superior de AUD en el Bajo. Las medias obtenidas fueron de 295, 256 y 244 mm en Bajo, Loma y Media Loma, respectivamente. Si bien no se halló efecto del tratamiento, las parcelas CCC presentaron reservas de AUD superiores a las SCC en todos los ambientes, con un máximo de 20 mm más en la Media Loma. 
En la etapa de inicio del llenado de granos (R5) del cultivo de soja (Figura 6), no se hallaron efectos de tratamiento ni ambiente. Todas las parcelas disminuyeron significativamente $(\mathrm{p}<0,05)$ sus niveles de AUD -a excepción del Bajo CCC- respecto de la medición anterior, realizada en floración del cultivo de soja. No obstante, las parcelas CCC mostraron mayores reservas de AUD que las parcelas SCC, con 22 mm más de agua en el perfil evaluado. Los contenidos de AUD oscilaron entre el 68 y el 94\% del AUM.

Los resultados hallados indican que, luego del secado de los CC, las parcelas CCC mostraron una importante mejora en la capacidad de almacenar el agua de las preci- pitaciones, respecto de las parcelas SCC. Esto se evidenció a través de la recuperación de las reservas de agua consumidas por los CC durante el barbecho previo a la siembra de la soja, y de niveles de AU más elevados en R1 y R5 de del cultivo de soja.

Ernst (2004) obtuvo una respuesta similar, cuando las lluvias ocurridas durante el barbecho previo a la siembra de un cultivo de verano, igualaron las reservas de agua de los tratamientos con y sin cubierta vegetal. Si bien en años de baja oferta hídrica, Carfagno et al. (2013b) observaron una tendencia hacia la conservación del agua en el suelo, luego de terminado el ciclo de los CC, y similar AU de éstos respecto al testigo sin CC. No obstante, Restovich y

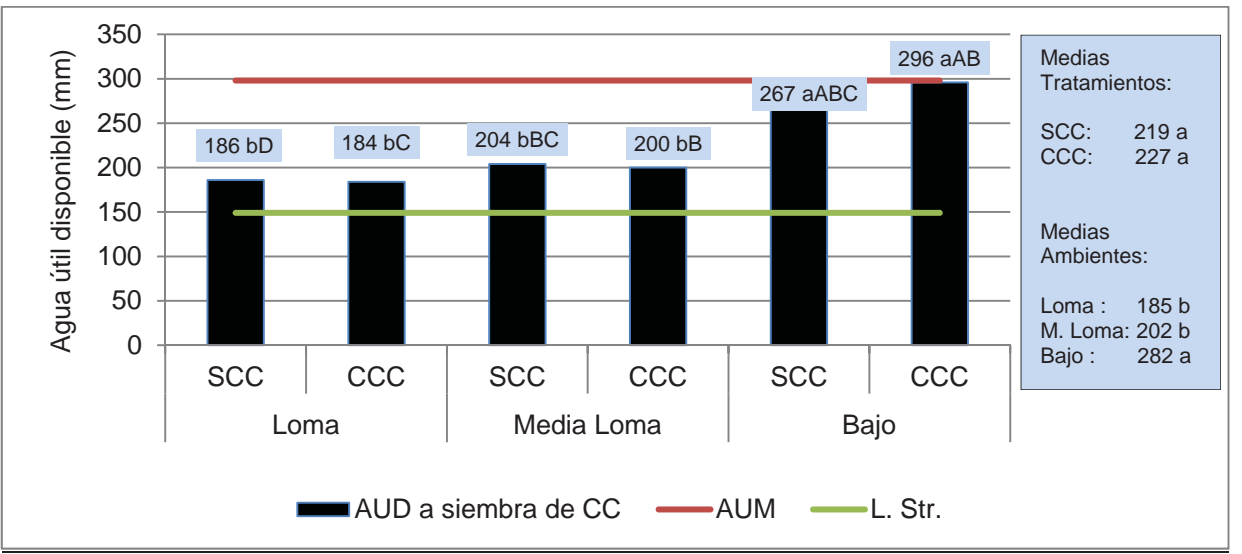

Figura 2. Lámina de agua útil disponible en milímetros, acumulada hasta 200 centímetros de profundidad a la siembra de los cultivos de cobertura. Ref.: AUD: Agua Útil Disponible; AUM: Capacidad Máxima de Agua Útil del Suelo; L.Str.: 50\% del Agua Útil Máxima; SCC: Sin Cultivos de Cobertura; CCC: Con Cultivos de Cobertura. Diferentes letras minúsculas entre barras indican diferencias significativas en cada momento de muestreo, distintas letras mayúsculas entre gráficos para cada TA, indican diferencias significativas entre fechas (test $F p<0,05$ ).

Figure 2. Sheet of useful water available in millimeters, accumulated up to 200 centimeters deep at planting of cover crops. Ref .: AUD: Available Useful Water; AUM: Maximum Useful Water Capacity of the Soil; L.Str.: 50\% of the Maximum Useful Water; SCC: No Cover Crops; CCC: With Cover Crops. Different lowercase letters between bars indicate significant differences at each sampling moment, different capital letters between graphs for each TA, indicate significant differences between dates (test $F p<0.05$ ). 
Andriulo (2013) analizaron las probables diferencias climáticas entre campañas y concluyeron que, considerando un escenario de lluvias primaverales por debajo del promedio histórico, el consumo de los CC registrado en los horizontes superficiales, podría afectar la producción del cultivo siguiente.

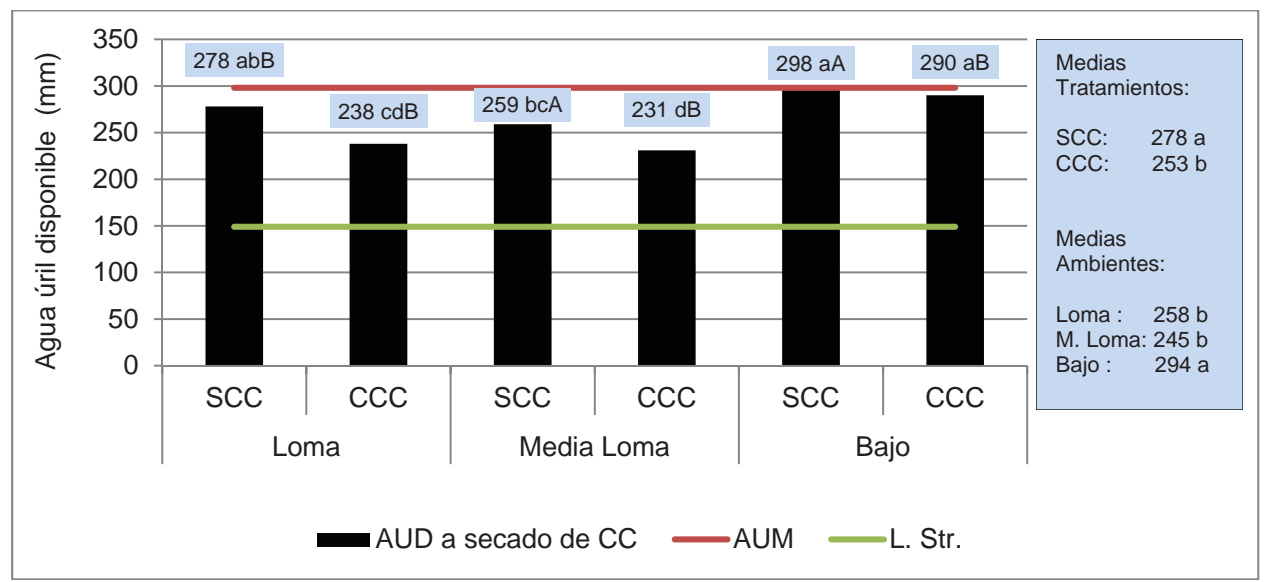

Figura 3. Lámina de agua útil disponible en milímetros, acumulada hasta 200 centímetros de profundidad al secado de los cultivos de cobertura. Ref.: AUD: Agua Útil Disponible; AUM: Capacidad Máxima de Agua Útil del Suelo; L.Str.: 50\% del Agua Útil Máxima; SCC: Sin Cultivos de Cobertura; CCC: Con Cultivos de Cobertura. Diferentes letras minúsculas entre barras indican diferencias significativas en cada momento de muestreo, distintas letras mayúsculas entre gráficos para cada TA, indican diferencias significativas entre fechas (test $F p<0,05$ ).

Figure 3. Sheet of useful water available in millimeters, accumulated up to 200 centimeters deep to drying of the cover crops. Ref .: AUD: Available Useful Water; AUM: Maximum Useful Water Capacity of the Soil; L.Str .: 50\% of the Maximum Useful Water; SCC: No Cover Crops; CCC: With Cover Crops. Different lowercase letters between bars indicate significant differences at each sampling moment, different capital letters between graphs for each TA, indicate significant differences between dates (test $F$ p <0.05). 


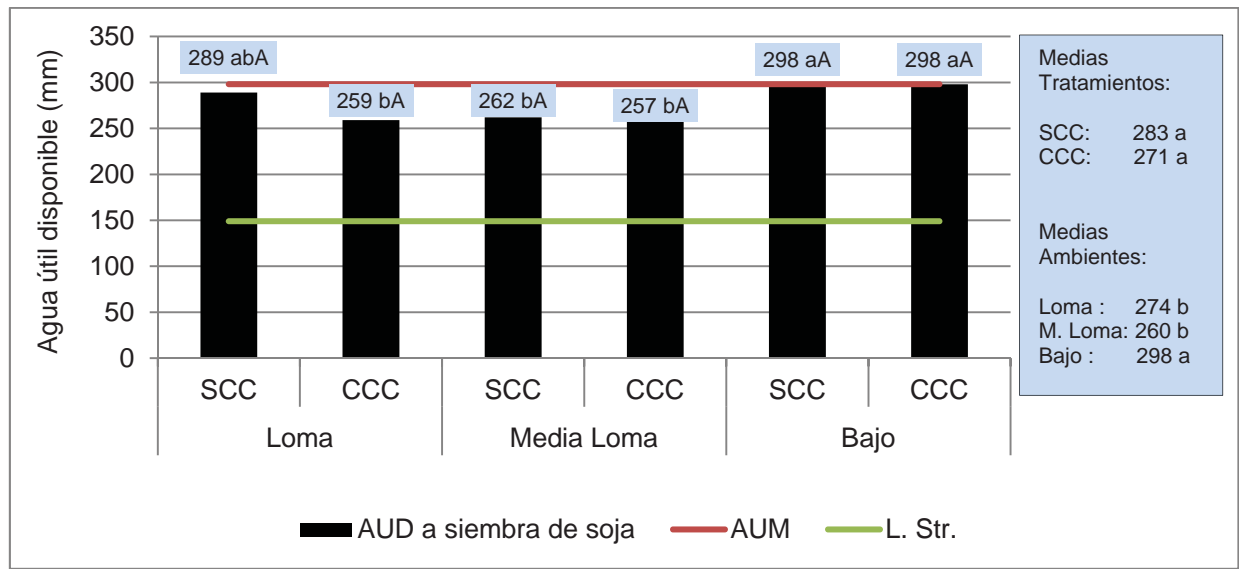

Figura 4. Lámina de agua útil disponible en milímetros, acumulada hasta 200 centímetros de profundidad a la siembra del cultivo de soja. Ref.: AUD: Agua Útil Disponible; AUM: Capacidad Máxima de Agua Útil del Suelo; L.Str.: 50\% del Agua Útil Máxima; SCC: Sin Cultivos de Cobertura; CCC: Con Cultivos de Cobertura. Diferentes letras minúsculas entre barras indican diferencias significativas en cada momento de muestreo, distintas letras mayúsculas entre gráficos para cada TA, indican diferencias significativas entre fechas (test $F p<0,05$ ).

Figure 4. Sheet of useful water available in millimeters, accumulated up to 200 centimeters deep when sowing the soybean crop. Ref .: AUD: Available Useful Water; AUM: Maximum Useful Water Capacity of the Soil; L.Str .: 50\% of the Maximum Useful Water; SCC: No Cover Crops; CCC: With Cover Crops. Different lowercase letters between bars indicate significant differences at each sampling moment, different capital letters between graphs for each TA, indicate significant differences between dates (test $F p<0.05$ ). 


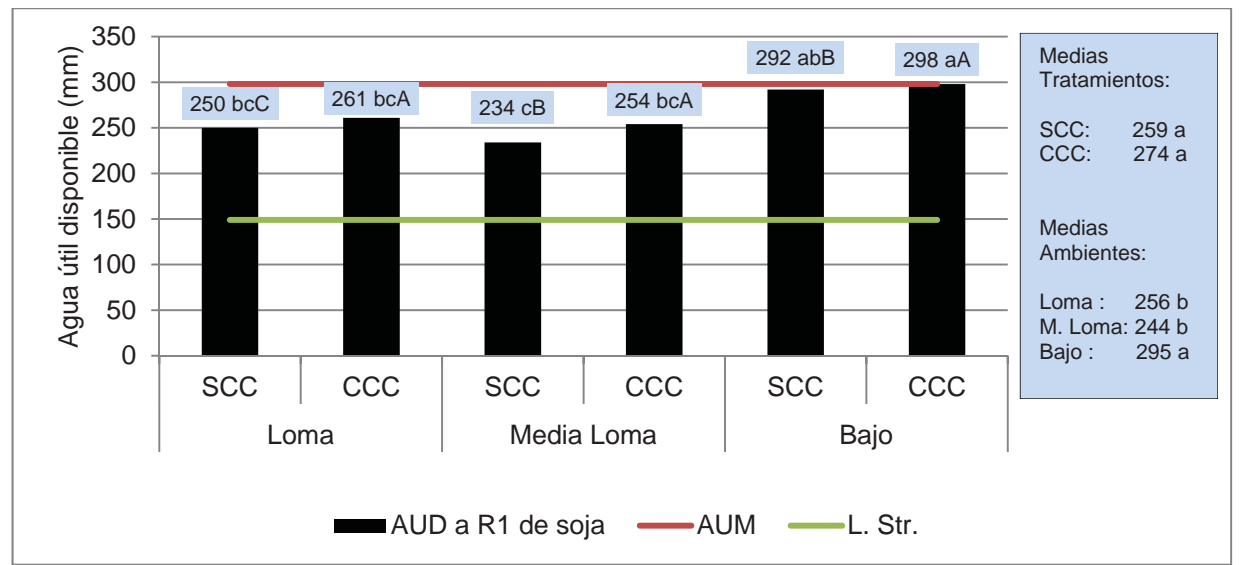

Figura 5. Lámina de agua útil disponible en milímetros, acumulada hasta 200 centímetros de profundidad a la floración del cultivo de soja. Ref.: AUD: Agua Útil Disponible; AUM: Capacidad Máxima de Agua Útil del Suelo; L.Str.: 50\% del Agua Útil Máxima; SCC: Sin Cultivos de Cobertura; CCC: Con Cultivos de Cobertura. Diferentes letras minúsculas entre barras indican diferencias significativas en cada momento de muestreo, distintas letras mayúsculas entre gráficos para cada TA, indican diferencias significativas entre fechas (test $F p<0,05$ ).

Figure 5. Sheet of useful water available in millimeters, accumulated up to 200 centimeters deep at flowering of the soybean crop. Ref .: AUD: Available Useful Water; AUM: Maximum Useful Water Capacity of the Soil; L.Str .: 50\% of the Maximum Useful Water; SCC: No Cover Crops; CCC: With Cover Crops. Different lowercase letters between bars indicate significant differences at each sampling moment, different capital letters between graphs for each TA, indicate significant differences between dates (test $F p<0.05$ ). 


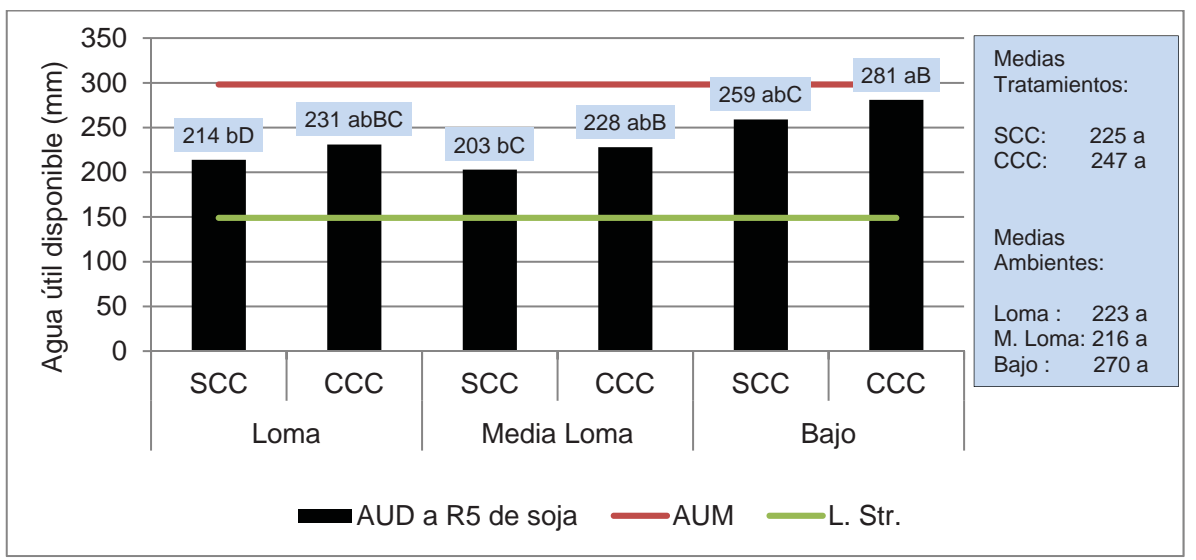

Figura 6. Lámina de agua útil disponible en milímetros, acumulada hasta 200 centímetros de profundidad, al inicio de llenado de granos del cultivo de soja. Ref.: AUD: Agua Útil Disponible; AUM: Capacidad Máxima de Agua Útil del Suelo; L.Str.: 50\% de Agua Útil Máxima; SCC: Sin Cultivos de Cobertura; CCC: Con Cultivos de Cobertura. Diferentes letras minúsculas entre barras indican diferencias significativas en cada momento de muestreo, distintas letras mayúsculas entre gráficos para cada TA, indican diferencias significativas entre fechas (test $F p<0,05$ ).

Figure 6. Sheet of useful water available in millimeters, accumulated up to 200 centimeters deep, at the beginning of the filling of grains of the soybean crop. Ref .: AUD: Available Useful Water; AUM: Maximum Useful Water Capacity of the Soil; L.Str .: 50\% of Maximum Useful Water; SCC: No Cover Crops; CCC: With Cover Crops. Different lowercase letters between bars indicate significant differences at each sampling moment, different capital letters between graphs for each $T A$, indicate significant differences between dates (test F $p<0.05$ ). 
Efecto sobre el rendimiento en granos del cultivo de soja: Los rendimientos de soja (Figura 7) sobre el CC mostraron una significativa interacción ambiente por tratamiento Los rindes del cultivo presentaron incrementos significativos en Media Loma CCC y Bajo CCC, con valores 19 y $10 \%$ mayores a Media Loma SCC y Bajo SCC, respectivamente. En Loma CCC, también hubo mayores rendimientos, pero no llegaron a ser significativos, respecto de los obtenidos en Loma SCC.

En cuanto al peso de mil granos (Tabla 3), el análisis de sus resultados mostró efectos altamente significativos de ambiente y tratamiento, sin interacción entre ambos. Las parcelas CCC tuvieron un $\mathrm{P}_{1000}$ promedio de $171 \mathrm{~g}$, significativamente superior $(\mathrm{p}<0,05)$ al $\mathrm{P}_{1000}$ de $167 \mathrm{~g}$ obtenido en las parcelas SCC. Por su parte, los ambientes de Loma, Bajo y Media Loma presentaron $\mathrm{P}_{1000}$ medios de 175, 168 y 163 g, respectivamente, todos con diferencias significativas entre sí. El análisis del NG (Tabla 4), detectó una interacción altamente significativa entre ambiente y tratamiento $(\mathrm{p}<0,05)$. La Media Loma CCC tuvo valores de NG significativamente superiores a la Media Loma SCC. Por otra parte, la Loma CCC y Bajo CCC también mostraron valores superiores a la Loma SCC y al Bajo SCC, pero en este caso las diferencias no fueron significativas.

En las parcelas CCC, los incrementos de rendimiento de soja, se explicaron por el aumento del $\mathrm{P}_{1000}$ y del NG del cultivo. Es probable que la mejora en el almacenaje de agua en ellas, haya incidido en la definición de estos componentes del rendimiento. El menor nivel de AUD en las parcelas SCC,

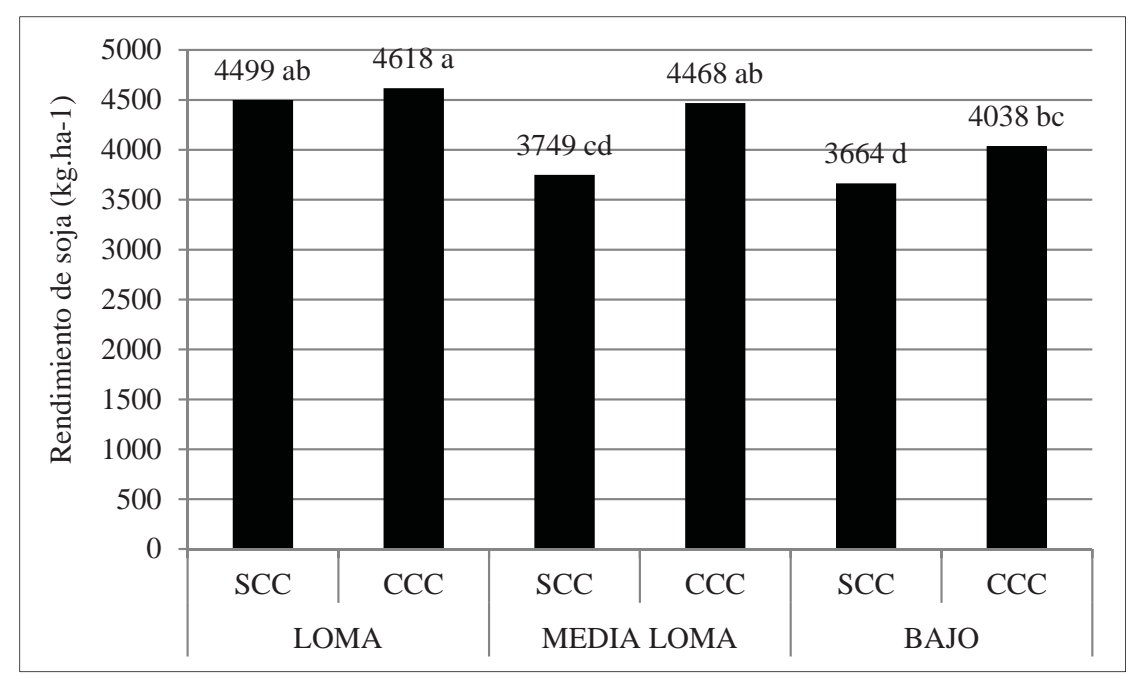

Figura 7. Rendimientos de soja en kilos por hectárea. Referencias: SCC: Sin Cultivos de Cobertura; CCC: Con Cultivos de Cobertura. Diferentes letras minúsculas, indican diferencias significativas (test $F$ p $<0,05$ ).

Figure 7. Soybean yields in kilos per hectare. References: SCC: No Cover Crops; CCC: With Cover Crops. Different lowercase letters indicate significant differences (test F $p<0.05$ ). 
pudo limitar una mayor fijación de granos. En las parcelas CCC, la mayor cantidad de AUD, posiblemente favoreció la producción de soja en una etapa considerada crítica para la determinación del rendimiento del cultivo.

Posiblemente, la respuesta positiva en rendimiento de soja a los CC en todos los ambientes evaluados, haya sido consecuencia del aumento del almacenaje de agua útil y de algunas propiedades del suelo -no presentadas en este trabajo lo que Ruffo y Parsons (2004) citados por Vanzolini et al. (2013) llaman "efecto rotación” y que posibilita el incremento de las diferencias entre tratamientos, ante aumentos de la fertilidad del suelo.

Rimski-Korsakov et al. (2015) evaluaron un gran conjunto de experiencias con CC, separando soja de maíz, en ambientes húmedos y semiáridos. Estos autores encontraron que en ninguno de estos cultivos se vio afectado significativamente su rendimiento en zonas húmedas, por la inclusión de un CC como antecesor. Tampoco la soja en zonas semiáridas muestra diferencias significativas, pero sí el maíz presenta en esta región rendimientos superiores por la inclusión de una leguminosa como CC antecesor. Por su parte, Restovich y Andriulo (2013) no encontraron diferencias en rendimiento de soja entre tratamientos con y sin CC en el año más húmedo y sí en el año más seco, lo que según estos autores estuvo posiblemente asociado al consumo de agua de estos antecesores y a la falta de precipitaciones que no recargaron el perfil del suelo.

La reserva de agua en la secuencia agrícola con la inclusión de un CC sigue siendo objeto de trabajos experimentales debido a las múltiples combinaciones entre factores naturales (precipitaciones, radiación, pendientes, tipos y estado de deterioro de suelos) y productivos (rotaciones, especies, densidades de siembra, forma, tipo y oportunidad de supresión de crecimiento) que muestran una gran diversidad de resultados en la región pampeana argentina.

Tabla 3. Peso de mil granos en gramos y número de granos por metro cuadrado del cultivo de soja. Table 3. Weight of thousand grains in grams and number of grains per square meter of the soybean crop.

\begin{tabular}{|c|c|c|c|}
\hline \multirow{2}{*}{ Ambiente } & \multirow{2}{*}{ Tratamiento } & $\mathrm{P}_{1000}$ & NG \\
\cline { 3 - 4 } & & $\mathrm{g}$ & granos. $\mathrm{m}^{-2}$ \\
\hline \multirow{2}{*}{ Loma } & $\mathrm{SCC}$ & $173 \mathrm{~b}$ & $2598 \mathrm{ab}$ \\
\cline { 2 - 4 } & $\mathrm{CCC}$ & $178 \mathrm{a}$ & $2600 \mathrm{ab}$ \\
\hline \multirow{2}{*}{ Media Loma } & $\mathrm{SCC}$ & $162 \mathrm{c}$ & $2308 \mathrm{c}$ \\
\cline { 2 - 4 } & $\mathrm{CCC}$ & $164 \mathrm{c}$ & $2723 \mathrm{a}$ \\
\hline \multirow{2}{*}{ Bajo } & $\mathrm{SCC}$ & $164 \mathrm{c}$ & $2231 \mathrm{c}$ \\
\cline { 2 - 4 } & $\mathrm{CCC}$ & $173 \mathrm{~b}$ & $2341 \mathrm{bc}$ \\
\hline
\end{tabular}

Referencias: SCC: sin cultivos de cobertura; CCC: con cultivos de cobertura. $P_{1000}:$ peso de mil granos; NG: número de granos por metro cuadrado. Letras distintas en sentido vertical indican diferencias significativas $(p<0,05)$. References: SCC: no cover crops; CCC: with cover crops. $P_{1000}$ : weight of thousand grains; NG: number of grains per square meter. Different letters vertically indicate significant differences $(p<0.05)$. 


\section{CONCLUSIONES}

El aporte de $\mathrm{C}$ al sistema a través de MS de los CC no presentó diferencias significativas entre los ambientes evaluados.

El aporte de $\mathrm{C}$ a partir de la producción de MS de hojas y tallos del cultivo de soja (soja 1), fue significativamente superior en las parcelas CCC respecto de las parcelas SCC.

El aporte de C a partir de la MS de vainas con granos del cultivo de soja (soja 2) fue significativamente superior en las parcelas CCC respecto de las parcelas SCC.

En el momento de secado de los CC, las parcelas CCC tuvieron significativamente menos AUD que las parcelas SCC.

En los momentos de siembra, floración e inicio de llenado de granos del cultivo de soja, las parcelas SCC y CCC no presentaron diferencias significativas en el AUD.

La soja tuvo rendimientos significativamente superiores en Media Loma CCC y Bajo CCC respecto de las parcelas SCC en esos ambientes, a través de un mayor NG y/o un superior $\mathrm{P}^{1000}$. En Loma CCC respecto de Loma SCC los incrementos de rendimientos no llegaron a ser significativos.

\section{BIBLIOGRAFÍA}

Carfagno, P.F., Eiza, M.J., Quiroga, A., Babinec, F., Chagas, C. y Michelena, R. 2013a. Agua disponible en monocultivo de soja con cultivos de cobertura y barbechos reducidos en la región semiárida y subhúmeda Pampeana. Ciencia del Suelo, 31 (1) 67-81.

Carfagno, P., Eiza, M., Babinec, F. y Quiroga, A. 2013b. Inclusión de cultivos de cobertura en la dinámica hídrica de Hapludoles y Haplustoles del oeste de la provincia de Buenos Aires y noreste de La Pampa.Contribuciones de los cultivos de cobertura a la sostenibilidad de los sistemas de producción. Ediciones INTA, 11: 83-87.

Caviglia, O. P., Sadras, V.O. y Andrade, F.H. 2004. Intensification of agricultura in the south-eastern pampas I. Capture and efficiency in the use of wáter and radiation in double cropped wheat-soybean.Field Crops Research, 87:117-129.

Caviglia, O.P.; Novelli, L.; Gregorutti V.C.; Van Opstal N.V. Y R.J.Melchiori. 2013. Cultivos de cobertura invernales: una alternativa de intesificación sustentable en el centro-oeste de Entre Ríos. Contribuciones de los cultivos de cobertura a la sostenibilidad de los sistemas de producción. Cap.18: 148-157. Ediciones INTA. ISBN 978-987-679-177-9.

Duval M.; Martinez J.M.; Iglesias, J.; Galantini, J.A. \& L. Wall. 2015. Secuencia de cultivos y su efecto sobre las fracciones orgánicas del suelo. El Impacto de los Sistemas actuales de cultivo sobre las Propiedades Químicas del Suelo y sus efectos sobre los Balances de Carbono. Pg.51-55. INTA Ediciones. Colección INVESTIGACIÓN, DESARROLLO E INNOVACIÓN. $1^{\text {a }}$ ed. Coronel Suarez, Buenos Aires. ISBN 978-987-521-624-2

Ernst, O. 2004. Leguminosas como cultivo de cobertura. Informaciones Agronómicas del Cono Sur. IPNI No21.

| Revista FAVE - Ciencias Agrarias 21 (1) 2022 
Fehr, W.R. \& C.E. Caviness. 1977. Stages of soybean development. Iowa St. Univ. Special Report 80. 11p.

INTA. 1988. Carta de Suelos de la República Argentina. Hoja 3360-13 y14. Cañada de Gómez y Rosario.

Lu, Y.C., Watkins, K.B. y Abdul-Baki, A.A. 2000. Cover crop in sustainable food production. Food Reviews International, 16: 121-157.

Melgares, E., Wingeyer, A., Ozust, J., Eclesia, R. \& Ocaranza B. 2018. Evolución del agua útil de un suelo molisol con diferentes cultivos de cobertura invernales. XXVI Congreso Argentino de la Ciencia del Suelo. Tucumán, Rep.Argentina.

Novillo, B., Voisin, A., Pellegrini, A., Chamorro, A., Bezus, R., Machetti, N. y Golik, S. 2018. Diferentes estrategias de barbecho en cultivos extensivos: efecto sobre propiedades químicas del suelo. XXVI Congreso Argentino de la Ciencia del Suelo. Tucumán, Rep. Argentina.

Quiroga, A.R., Funaro, D.O., Fernández,R. y Noellemeyer, E. J. 2005. Factores edáficos y de manejo que condicionan la eficiencia del barbecho en la región pampeana.Ciencia del Suelo, 23 (1), 79-86.
Restovich, S. y Andriulo, A. 2013. Cultivos de cobertura en la rotación soja-maíz: biomasa aérea, captura de nitrógeno, consumo de agua y efecto sobre el rendimiento en grano. Contribuciones de los cultivos de cobertura a la sostenibilidad de los sistemas de producción. Ediciones INTA, 5: 29-35.

Rimski-Korsakov, H., Alvarez, C.R. y Lavado, R.S.2015. Cover crops in the agricultural systems of the Argentine Pampas. Journal of Soil and Water Conservation, 70(6), 134140.

Ruffo, M.L. y Parsons, A.T. 2004. Cultivos de cobertura en sistemas agrícolas. Informaciones Agronómicas del Cono Sur, IPNI No 21.

Siqueira Leite, M.H.; Guimarães Couto, E.; Silva Amorim, R.S.; Lenza Da Costa, E. \& L. Maraschin. 2009. Perdas de solo e nutrientes num latossolo vermelho-amarelo ácrico típico, com diferentes sistemas de preparo e sob chuva natural. R. Bras. Ci. Solo, 33:689-699. Thornthwaite, C.W. \& J.R. Mather. 1955. The wáter balance: Centerton, N.J., Laboratory of Climatology, Publications in Climatology, v.8, No1, p.1-104.

Vanzolini, J.I.; Galantini, J. Y R. Agamennoni. 2013. Cultivos de cobertura de Vicia villosa Roth. en el valle bonaerense del Río Colorado. Contribuciones de los cultivos de cobertura a la sostenibilidad de los sistemas de producción. Cap.4: 21-28. Ediciones INTA. ISBN 978-987-679-177-9 